\title{
The patient safety collaborative programme: opportunities for physician engagement
}

\author{
Authors: John Illingworth, ${ }^{A}$ Cheryl Crocker ${ }^{B}$ and $C$ Michael Roberts ${ }^{C}$
}

Driving improvements in patient safety has been a core goal of the Academic Health Science Networks (AHSNs) in England since their inception in 2013. The National Patient Safety Collaborative Programme, nested within the 15 geographically located AHSNs, was established in 2014 in response to the Berwick review. In 2019, the new NHS national patient safety strategy was published, which placed the AHSNs as a key vehicle for delivering its ambitions. This paper explores the achievements of, and opportunities presented by, the collaborative in addressing some of the key patient safety challenges facing physicians and their wider teams. Case studies illustrate the AHSNs' contribution to support national ambitions, including the adoption of the National Early Warning Score (NEWS) 2, and the impact of regionally-led work on patient outcomes, such as reducing mortality from sepsis and acute kidney injury. We set out current activities, opportunities for physician engagement and plans for future work.

KEYWORDS: Patient safety, quality improvement, innovation adoption, networks

DOI: 10.7861/clinmed.2019-0498

\section{Background}

In 2012, the report Innovation, health and wealth highlighted the need to accelerate the adoption of innovation in the NHS. ${ }^{1}$ In 2013, 15 Academic Health Science Networks (AHSNs) were established across England to help meet this ambition, to spread innovation at pace and scale, improve health and generate economic growth. Each AHSN works across a distinct geography, connecting NHS and academic organisations, local authorities, the third sector and industry to facilitate change across whole health and social care economies to improve outcomes for patients. Funded by

\footnotetext{
Authors: ${ }^{A}$ head of patient safety, UCLPartners, London, UK and honorary research associate, Institute of Global Health Innovation, London, UK; ${ }^{B}$ patient safety director, Academic Health Science Network, UK and honorary associate professor, University of Nottingham, Nottingham, UK; ' managing director, UCLPartners, London, UK, professor of medical education for clinical practice, Queen Mary University of London, London, UK and consultant physician, The Princess Alexandra Hospital, Harlow, UK
}

NHS England, NHS Improvement and the Office for Life Sciences, a diverse range of programmes and activities provide a unique opportunity for physician engagement; one of these programmes is the Patient Safety Collaborative (PSC), which was established to accelerate improvements in patient safety across England.

\section{Emergence of patient safety}

Patient safety is a relatively recent phenomenon. As recently as 25 years ago, harm caused by healthcare, such as healthcareacquired infections, was generally regarded as an inevitable consequence of healthcare. ${ }^{2}$ Today, such problems are seen as both unacceptable and preventable, and it is this intolerance of harm that has driven a number of significant improvements and achievements in patient safety. For instance, due to the concerted efforts of staff and the impetus provided by national campaigns, cases of methicillin-resistant Staphylococcus aureus and Clostridium difficile decreased dramatically between 2007-2010. ${ }^{3}$

However, advances in technology, evolution of our understanding of harm, ever-increasing expectations from patients and the drive for care to be delivered in non-clinical settings and by patients and carers themselves, means that patient safety is never finished; it is a never-ending pursuit, where standards continue to rise but where more needs to be done. There is a growing feeling that there needs to be a step-change in how safety improvement is approached to make further improvements. ${ }^{4}$

\section{The PSC programme}

In 2013, a national review of patient safety in England was conducted by Prof Don Berwick in the light of the failings at Mid Staffordshire NHS Foundation Trust. ${ }^{5}$ The Department of Health and Social Care responded to a recommendation in the Berwick review to establish regional expertise that could help NHS organisations build capacity and capability to improve patient safety. ${ }^{6}$ In October 2014, the PSC programme was launched, with the following underpinning principles:

\footnotetext{
$>$ securing local engagement through structured quality improvement (QI) initiatives leading towards transformational change

> building system-wide capability for staff and patients in quality and safety improvement

> spreading QI interventions both within and across AHSN regions

$>$ actively contributing to national sharing and learning.
}

Each of the 15 regional PSCs, hosted by their respective AHSN, was encouraged to build upon existing initiatives or instigate new 
areas of work in areas identified to be of local importance, using a range of QI tools and techniques. In 2017, the work of each regional PSC was realigned around a smaller number of national priorities to focus efforts and achieve greater impact in the following areas:

$>$ fostering a positive safety culture

$>$ improving the identification and management of deteriorating patients (Box 1)

> improving safety in maternal and neonatal services.

\section{Using QI to improve patient safety}

The work of the PSC is underpinned by a methodological approach to use QI as part of collaborative structures to drive continuous improvements in patient safety. There is no single definition of QI, nor a single way to approach it. ${ }^{13}$ Drawn from techniques in other industries, the Institute for Healthcare Improvement's (IHI's) Model for Improvement is perhaps the most commonplace in healthcare and is used extensively as part of the PSC. ${ }^{14}$ It is not a panacea, but it has the advantage of enabling frontline teams to use practical

\section{Box 1. Improving the identification and management of deteriorating patients}

The mismanagement of deterioration is the most common area of systemic failure of avoidable patient death across the NHS, while poor communication is the leading root cause of adverse events in healthcare. ${ }^{7}$ In recent years, the regional Patient Safety Collaboratives (PSCs) have been working with acute and non-acute teams to support improvements in the identification and management of deteriorating patients. The work has been supported through a combination of locally-led activities, national coordinated work as well as a range of national policy levers to support change.

\section{Supporting local implementation of national policy: embedding the use of the National Early Warning Score $\mathbf{2}$}

The National Early Warning Score 2 (NEWS2) is the tool endorsed by NHS England and NHS Improvement to identify acutely ill patients, including those with sepsis, in hospitals in England. It originated out of a working party convened by the Royal College of Physicians (RCP), with the original NEWS tool released in $2012 .^{8}$ It is a simple aggregate scoring system in which a score is allocated to six physiological measurements already recorded in routine practice (respiratory rate, oxygen saturation and so on), where the magnitude of the score reflects the degree to which the parameter varies from the norm. ${ }^{9}$

The regional PSCs have been supporting acute and ambulance trusts to implement NEWS2 so that it becomes standardised across the acute sector and creates a common language when quantifying patient deterioration in handovers of care. Support has typically been in the form of creating multidisciplinary and interorganisational networks, running regional NEWS2 events and working alongside individual hospitals to overcome specific implementation challenges, most commonly around integration with electronic systems, supporting the use of structured communication tools (such as Situation, Background, Assessment and Recommendation (SBAR)) to communicate critical information, and establishing escalation protocols to ensure the timely and appropriate medical response to deterioration.

\section{Implementing a system-wide approach to NEWS2}

The West of England PSC covers community and primary care, seven hospital sites, two mental health trusts, the ambulance service and, at the time of implementation, seven commissioners across the region. In 2015, the PSC began a programme of work to implement NEWS across the entire healthcare system, later supporting the system to transition to NEWS2 when it was released. The vision was that, regardless of the setting, an acutely unwell patient has a NEWS score calculated at the point of assessment and then at every handover of care to ensure that the action taken means that the patient is seen at the right time in the right place by the right grade of clinician.

The PSC used a quality improvement collaborative approach to bring together staff from across the health system every 6 months, while each area brought together local teams to meet more frequently to address specific challenges for their region. The PSC also facilitated the introduction of NEWS into the South Western Ambulance Service NHS Foundation Trust electronic patient care record and helped with implementation and spread. The work has resulted in all acute trusts, out of hours general practitioner services, mental health trusts, community service providers and clinical commissioning groups signing up to support the adoption and spread of NEWS2, and communicating the score at the interfaces of care across the system. ${ }^{10}$ Research found that, between April 2016 and November 2017. $63 \%$ of over 1 million attendances by the ambulance service in the region had a NEWS recorded. ${ }^{11}$

\section{Reducing mortality from sepsis and acute kidney injury across London and the south-east of England}

In 2014, UCLPartners - the Academic Health Science Network which serves the population of north-east and north-central London and parts of three connecting home counties - brought together senior clinicians and hospital leaders from its NHS trust partners to identify clinical areas for a quality improvement programme in patient care and safety. Causing around 80,000 deaths annually in England - and the focus of national campaigns and influential reports - acute kidney injury (AKI) and sepsis emerged as urgent priorities for improvement.

Two collaboratives were established, bringing together professionals to learn from and motivate each other to improve care. Teams from 12 NHS trusts formed the sepsis collaborative, and teams from nine trusts formed the AKI collaborative. Both completed three main programme phases - baseline, improvement and implementation - from September 2015 to June 2017. Throughout these phases, teams took part in a series of group learning sessions intersected with action periods during which they tested and measured improvements in practice in their own hospital settings. Interventions included redesigned care pathways, creation of dedicated roles and awareness campaigns.

In total, records from 950 AKI and almost 1,200 sepsis cases were included in the final analysis. Both collaboratives exceeded their initial aims for improving survival rates: AKI deaths reduced by $47 \%$, and sepsis deaths fell by $24 \%$. In sepsis, patients' median length of stay in hospital was reduced by $20 \%$ and admissions to intensive care were cut by $52 \%$. In AKI, rates of kidney function recovery were increased by $19.5 \%$. Both collaboratives also demonstrated improvements in clinical processes, such as timely recognition of the conditions, improved documentation and best practice follow-up (see supplementary material S1). ${ }^{12}$ 
techniques to understand the nature and scale of the problem being tackled, introduce small tests of changes to address the problem, and measure the impact of those changes in almost real time.

Utilising the IHI methodology, the PSC makes particular use of the QI collaborative method, which is an organised, multifaceted approach involving teams from multiple healthcare sites to learn, apply and share improvement methods, ideas and data in a given area or topic. ${ }^{15}$ Teams are supported by a central team who provide additional coaching to help teams apply QI methods in their own work environment, and to measure the effect of the changes introduced.

Such approaches are based on the principle that healthcare teams can make changes more quickly and more effectively when working together, rather than in silos. This can be for several reasons: first, because improvements in safety and quality often necessitate multidisciplinary or multi-agency engagement, for instance, if trying to reduce emergency admissions to hospital by equipping community teams to better identify and manage deteriorating patients; second, because it is often the case that a safety or quality challenge faced by one healthcare team has already been tackled by another; third, because collaborative approaches can enable change to be made on a larger-scale; fourth, because they can facilitate the standardisation of practice where appropriate, such as in the adoption of improvement interventions like care bundles and checklists; and fifth, because they can help to engage clinical teams at a regional level to support the implementation of national policies and initiatives.

QI collaboratives are, however, not without their challenges. Like other change or improvement methodologies, their effectiveness relies on a number of social, technical and contextual factors. Dixon-Woods and Martin also identified that, to maximise the effectiveness of QI efforts, healthcare needs to act more like a system rather than hospital-by-hospital or care home-by-care home. The PSC has embraced this through its work to standardise the approach to, and develop a common language for, the identification and management of deteriorating patients (see Box 1), complemented by having a common aim and carrying out work to build the skills of staff to implement and measure change (see supplementary material S2). ${ }^{16}$

A core advantage to the collaborative approach is that it allows highly pressured frontline teams to come together, giving them the necessary time, space and support to plan and deliver improvement work. This is most effective when individuals have explicit support from a senior colleague and protected time to participate, something which is increasingly challenging given the current pressures on the health service, even though taking part in quality improvement activities to improve safety is now explicitly required within Good medical practice. ${ }^{17}$

\section{The NHS patient safety strategy}

In July 2019, a new strategy (The NHS patient safety strategy) was launched. ${ }^{18}$ The strategy describes how the NHS will continuously improve patient safety by:

> improving understanding of safety by drawing intelligence from multiple sources of patient safety information (insight)

> equipping patients, staff and partners with the skills and opportunities to improve patient safety throughout the whole system (involvement)

> designing and supporting programmes that deliver effective and sustainable change in the most important areas (improvement).
The PSC has been identified as the main vehicle through which to deliver the improvement section of the strategy, with each regional PSC making use of its position as a convenor and connector in the system within which it operates.

\section{Local efforts can have a national impact}

The traditional way of trying to achieve change at scale within healthcare has invariably involved a top-down approach, with mixed results. ${ }^{19}$ The PSC programme has benefitted from national policy drivers to support change in areas, such as the introduction of NEWS2 (see Box 1), ranging from financial incentives to issuing patient safety alerts. However, frontline teams have benefited from having a regional PSC to act as a buffer to these directions, providing improvement support to help teams meet their obligations and creating opportunities for clinicians to engage with peers from other organisations to share their learning and knowledge.

\section{Current and future programmes of work: opportunities for physician involvement}

The PSC programme consists of the following current and upcoming programmes of work. They are multiprofessional in nature, but rely heavily on physician engagement in both the planning, design, delivery and wider adoption of the interventions being implemented.

\section{> Improving the identification and management of} deteriorating patients: which includes the recognition, communication and escalation of deterioration across all sectors and specialties. Future work will focus on supporting the adoption of NEWS2 right across the health and care system.

> Improving safety in maternity and neonatal care: this programme supports the NHS 'better births' programme, which has the ambition to reduce harm by $50 \%$ by $2025 .{ }^{20}$ All units in England have access to PSC-facilitated networks, known as local learning systems, to bring clinicians, mothers and system leaders together to drive improvements across local regions.

> Improving the safety of medicines administration in care homes: this programme will support improvements in the administration of medicines in care homes, a significant source of risk for our elderly and frail population. This will require the support of clinicians from hospital to the community in the review of the medicine burden.

\section{> Supporting the adoption and spread of tested patient} safety interventions: each regional PSC is supporting local clinical teams to implement, and drive more effective use of, a number of improvement interventions. These are:

> emergency department (ED) patient safety checklist: a time-based framework outlining the clinical tasks that need completing for each patient in the first four hours of their admittance to the ED

$>$ Emergency Laparotomy Collaborative: supporting adoption of an evidence-based set of interventions to improve outcomes for patients

> Prevention of Cerebral Palsy in the Preterm Infant (PReCePT): reducing the incidence of cerebral palsy by offering magnesium sulphate to eligible women during preterm labour

> chronic obstructive pulmonary disease hospital discharge bundle (see Box 2). 
Box 2. Improving use of the chronic obstructive pulmonary disease hospital discharge bundle

Chronic obstructive pulmonary disease (COPD) accounts for around 10\% of emergency hospital medical admissions in the UK, totalling 90,000 patients a year. ${ }^{21}$ Around one-quarter of these patients are re-admitted to hospital within 30 days, rising to $43 \%$ within 90 days. ${ }^{22}$ To support improvements in the care of COPD patients admitted to hospital with an acute exacerbation, and to reduce the risk of readmission to hospital, the British Thoracic Society (BTS) developed and piloted a hospital discharge care bundle. ${ }^{23}$ The BTS Bundle consists of the following components, which research found to be associated with a reduction in the 30-day readmission rate: ${ }^{24}$

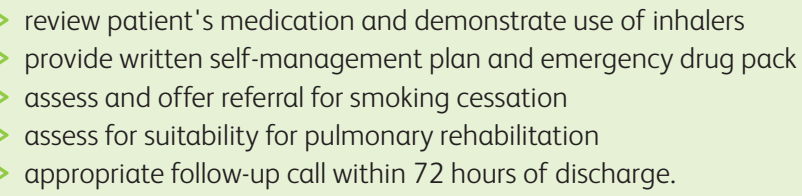

In 2019, each regional PSC began working with its local hospital to support the effective and consistent implementation of the bundle. Hospitals have been provided with a wide range of support including access to networks to share best practice, provision of data dashboards to show progress with uptake of the bundle, and coaching in quality improvement skills to enable teams to initiate local improvement work. Regional PSCs have also supported hospitals to improve the accuracy of the data reported through the National Asthma and COPD Audit Programme (NACAP). This work is ongoing, and acute and non-respiratory physicians, particularly care of the elderly physicians, are especially encouraged to participate given the challenge of ensuring uptake of the bundle for patients under their care.

\section{Conclusion}

Physicians have played, and continue to play, a significant role in the planning and delivery of the national PSC programme. A number of programmes selected for national spread and adoption were developed by clinicians who identified a solution to a significant and common local patient safety challenge, often using quality improvement techniques to iterate the solution. The PSC programme has achieved its successes in part by relying on a pipeline of ideas and innovations coming from frontline teams, with each regional PSC ensuring that there continues to be appropriate networks and capability-building opportunities to allow clinicians to incubate and develop these ideas into practical improvements with the potential for scale. The PSC programme has used the existing evidence together with new learning, particularly around dovetailing appropriate national levers with a regional system to engage, support and bring the best out of its local clinical teams, to optimise implementation of safety initiatives at pace and scale within the NHS.

The PSC provides a unique opportunity for physicians to be involved locally, regionally and nationally. Each regional PSC requires support from clinical leads to help lead the multiple programmes they are commissioned to deliver. Physicians have a valuable part to play locally in developing and testing innovations, feeding back successes and challenges which inform the programmes and feed up to national teams, which can in turn influence national policy.

\section{Summary of key practice implications}

> Physicians should contact their local AHSN's PSC to discover opportunities to develop and test patient safety innovations, and lead or participate in large-scale patient safety improvement programmes across a range of clinical areas spanning entire health and care systems.

> The PSCs based within the 15 AHSNs can be contacted at www. ahsnnetwork.com/contact-the-ahsn-network.

$>$ The information about current and future programmes of work for the PSC was correct at the time of writing but subject to change given the emerging COVID-19 crisis. The programme now includes work to improve the safety of tracheostomy care. For further information, please visit www.tracheostomy.org.uk.

\section{Supplementary material}

Additional supplementary material may be found in the online version of this article at www.rcpjournals.org/clinmedicine:

S1 - Summary of the UCLPartners sepsis and acute kidney injury improvement collaborative 2015-2017.

S2 - Patient safety collaborative activity between September 2016 and December 2018.

\section{Acknowledgements}

NHS England and NHS Improvement are acknowledged as the commissioners and funders of the national Patient Safety Collaborative programme since its inception. Thank you to Kevin Hunter, Nathalie Delaney and Dr Swapna Mandal for their input into the manuscript.

\section{References}

1 Department of Health, NHS Improvement. Innovation, health and wealth: Accelerating adoption and diffusion in the NHS. NHS, 2011. https://webarchive.nationalarchives.gov.uk/20130107013731/ http://www.dh.gov.uk/en/Publicationsandstatistics/Publications/ PublicationsPolicyAndGuidance/DH_131299 [Accessed 13 December 2019].

2 Vincent C, Amalberti R. Safer healthcare: Strategies for the real world. London: Springer, 2016.

3 Public Health England. Results from the mandatory surveillance of MRSA bacteraemia. Public Health England, 2014. https:// webarchive.nationalarchives.gov.uk/20140714025116/ http://www.hpa.org.uk/web/HPAweb\&HPAwebStandard/ HPAweb_C/1233906819629 [Accessed 13 December 2019].

4 Berwick D. In: Illingworth J. Continuous improvement of patient safety: The case for change in the NHS. London: The Health Foundation, 2015. 
5 National Advisory Group on the Safety of Patients in England. A promise to learn - a commitment to act. Improving the safety of patients in England. GOV.UK, 2013. www.gov.uk/government/ publications/berwick-review-into-patient-safety [Accessed 13 December 2019].

6 Department of Health. Hard truths: The journey to putting patients first. London: The Stationery Office, 2014.

7 Inada-Kim M. NEWS2: supporting the physician's role in leading on the deteriorating patient. Royal College of Physicians, 2018. www. rcplondon.ac.uk/news/news2-supporting-physician-s-role-leadingdeteriorating-patient [Accessed 13 December 2019].

8 Royal College of Physicians. National Early Warning Score (NEWS): standardising the assessment of acute-illness severity in the NHS. Report of a working party. London: RCP, 2012.

9 Royal College of Physicians. National Early Warning Score (NEWS) 2. London: RCP, 2017. www.rcplondon.ac.uk/projects/outputs/nationalearly-warning-score-news-2 [Accessed 13 December 2019]

10 West of England Academic Health Science Network. Spreading the NEWS: Collaborative working across the west of England to help spread the implementation of the National Early Warning Score (NEWS) to identify the deteriorating patient. WEAHSN, 2017. www.weahsn.net/wp-content/uploads/NEWS-Poster-FINALES-24-April-17.pdf [Accessed 13 December 2019].

11 Scott LJ, Redmond NM, Garrett ] et al. Distributions of the National Early Warning Score (NEWS) across a healthcare system following a large-scale roll-out. Emerg Med J 2019;36:287-92.

12 UCLPartners. The UCLPartners acute kidney injury (AKI) and sepsis patient safety collaboratives. London: UCLPartners, 2018.

13 Dixon-Woods M. How to improve healthcare improvement. BMJ 2019;367:15514.

14 Institute for Healthcare Improvement. How to improve. IHI. www. ihi.org/resources/Pages/HowtoImprove/default.aspx [Accessed 13 December 2019].

15 Wells S, Tamir O, Gray ] et al. Are quality improvement collaboratives effective? A systematic review. BMJ Qual Saf 2018;27:226-40
16 Dixon-Woods M, Martin GP. Does quality improvement improve quality? FHJ 2016;3:191-4.

17 General Medical Council. Good medical practice. Manchester: GMC, 2019. www.gmc-uk.org/ethical-guidance/ethical-guidance-fordoctors/good-medical-practice [Accessed 13 December 2019].

18 NHS England, NHS Improvement. The NHS patient safety strategy: Safer culture, safer systems, safer patients. NHS, 2019. https:// improvement.nhs.uk/documents/5472/190708_Patient_Safety_ Strategy_for_website_v4.pdf [Accessed 13 December 2019].

19 Dixon-Woods M, Leslie M, Tarrant C et al. Explaining Matching Michigan: an ethnographic study of a patient safety program. Implement Sci 2013;8:70.

20 The National Maternity Review. Better births: Improving outcomes of maternity services in England: A five year forward view for maternity care. The National Maternity Review. www.england.nhs. uk/wp-content/uploads/2016/02/national-maternity-review-report. pdf [Accessed 13 December 2019].

21 Stone RA, Holzhauer-Barrie J, Lowe D et al. National Chronic Obstructive Pulmonary Disease (COPD) Audit Programme: Who cares matters. London: Royal College of Physicians, 2015.

22 National Asthma and Chronic Obstructive Pulmonary Disease Audit Programme, Royal College of Physicians. Outcomes of patients included in the 2017 COPD clinical audit. London: RCP, 2019.

23 Calvert J, Lim WS, Rodrigo Turner A, Welham S. British Thoracic Society Pilot Care Bundle Project: A care bundles-based approach to improving standards of care in chronic obstructive pulmonary disease and community acquired pneumonia. BTS Reports 2014;6:4.

24 Hopkinson NS, Englebretsen C, Cooley N et al. Designing and implementing a COPD discharge care bundle. Thorax 2012;67:90-2.

Address for correspondence: Mr John H Illingworth, UCLPartners, 170 Tottenham Court Rd, Fitzrovia, London W1T 7HA, UK.

Email: john.illingworth@uclpartners.com 